\title{
POLYNOMIAL INEQUALITIES, MAHLER'S MEASURE, AND MULTIPLIERS
}

\author{
IGOR E. PRITSKER
}

\begin{abstract}
We survey polynomial inequalities obtained via coefficient multipliers, for norms defined by the contour or the area integrals over the unit disk. Special attention is devoted to the Szegó composition and the inequalities related to Mahler's measure.

We also consider a new height on polynomial spaces defined by the integral over the normalized area measure on the unit disk. This natural analog of Mahler's measure inherits many nice properties such as the multiplicative one. However, this height is a lower bound for Mahler's measure, and it fails an analog of Lehmer's conjecture.
\end{abstract}

\section{The Szegő COMPosition And POlynomial INEQUALITIES}

This paper is a survey of results on polynomial inequalities obtained via coefficient multipliers, and other topics related to Mahler's measure. Let $\mathbb{C}_{n}[z]$ and $\mathbb{Z}_{n}[z]$ be the sets of all polynomials of degree at most $n$ with complex and integer coefficients respectively. Mahler's measure of a polynomial $P_{n} \in \mathbb{C}_{n}[z]$ is defined by

$$
M\left(P_{n}\right):=\exp \left(\frac{1}{2 \pi} \int_{0}^{2 \pi} \log \left|P_{n}\left(e^{i \theta}\right)\right| d \theta\right) .
$$

It is also known as the contour geometric mean or as the $H^{0}$ Hardy space norm. The latter name is explained by the following relation to the Hardy spaces. Defining the Hardy space norm by

$$
\left\|P_{n}\right\|_{H^{p}}:=\left(\frac{1}{2 \pi} \int_{0}^{2 \pi}\left|P_{n}\left(e^{i \theta}\right)\right|^{p} d \theta\right)^{1 / p}, \quad 0<p<\infty,
$$

we note [18] that $M\left(P_{n}\right)=\lim _{p \rightarrow 0+}\left\|P_{n}\right\|_{H^{p}}$. An application of Jensen's inequality immediately gives that

$$
M\left(P_{n}\right)=\left|a_{n}\right| \prod_{\left|z_{j}\right|>1}\left|z_{j}\right|
$$

2000 Mathematics Subject Classification. Primary 11C08; Secondary 11G50, 30C10.

Key words and phrases. Polynomials, Mahler's measure, heights, zero distribution, Bergman spaces, inequalities, Szegö composition, approximation by polynomials with integer coefficients.

Research was partially supported by the National Security Agency under grant H9823006-1-0055, and by the Alexander von Humboldt Foundation. 
for $P_{n}(z)=a_{n} \prod_{j=1}^{n}\left(z-z_{j}\right) \in \mathbb{C}_{n}[z]$.

For a polynomial $\Lambda_{n}(z)=\sum_{k=0}^{n} \lambda_{k}\left(\begin{array}{l}n \\ k\end{array}\right) z^{k} \in \mathbb{C}_{n}[z]$, define the Szegö composition with $P_{n}(z)=\sum_{k=0}^{n} a_{k} z^{k} \in \mathbb{C}_{n}[z]$ by

$$
\Lambda P_{n}(z):=\sum_{k=0}^{n} \lambda_{k} a_{k} z^{k}
$$

If $\Lambda_{n}$ is a fixed polynomial, then $\Lambda P_{n}$ is a multiplier (or convolution) operator acting on $P_{n}$. More information on the history and applications of this composition may be found in [10], [1], [2] and [33]. De Bruijn and Springer [10] proved a very interesting general inequality stated below.

Theorem 1.1. Suppose that $P_{n} \in \mathbb{C}_{n}[z]$. If $\Lambda_{n} \in \mathbb{C}_{n}[z]$ and $\Lambda P_{n} \in \mathbb{C}_{n}[z]$ are defined by (1.1), then

$$
M\left(\Lambda P_{n}\right) \leq M\left(\Lambda_{n}\right) M\left(P_{n}\right) .
$$

If $\Lambda_{n}(z)=(1+z)^{n}$ then $\Lambda P_{n}(z) \equiv P_{n}(z)$ and $M\left(\Lambda_{n}\right)=1$, so that $(1.2)$ turns into equality, showing sharpness of Theorem 1.1. This result has not received the attention it truly deserves. In particular, it contains the following inequality that is usually attributed to Mahler, who proved it later in [26].

Corollary 1.2. $M\left(P_{n}^{\prime}\right) \leq n M\left(P_{n}\right)$

To see this, just note that if $\Lambda_{n}(z)=n z(1+z)^{n-1}=\sum_{k=0}^{n} k\left(\begin{array}{l}n \\ k\end{array}\right) z^{k}$, then $\Lambda P_{n}(z)=z P_{n}^{\prime}(z)$ and $M\left(\Lambda_{n}\right)=n$. Furthermore, (1.2) immediately answers a question about a lower bound for Mahler's measure of derivative raised in [14, pp. 12 and 194]. Following Storozhenko [42], we consider $P_{n}^{\prime}(z)=\sum_{k=0}^{n-1} a_{k} z^{k}$ and write

$$
\frac{1}{z}\left(P_{n}(z)-P_{n}(0)\right)=\sum_{k=0}^{n-1} \frac{a_{k}}{k+1} z^{k}=\Lambda P_{n}^{\prime}(z),
$$

where

$$
\Lambda_{n-1}(z)=\sum_{k=0}^{n-1} \frac{1}{k+1}\left(\begin{array}{c}
n-1 \\
k
\end{array}\right) z^{k}=\frac{(1+z)^{n}-1}{n z} .
$$

The result of de Bruijn and Springer (1.2) gives

Corollary 1.3. [42] We have $M\left(P_{n}(z)-P_{n}(0)\right) \leq c_{n} M\left(P_{n}^{\prime}\right)$, where

$$
c_{n}:=\frac{1}{n} M\left((1+z)^{n}-1\right)=\frac{1}{n} \prod_{n / 6<k<5 n / 6} 2 \sin \frac{k \pi}{n} .
$$

We note that $c_{n} \approx(1.4)^{n}$ as $n \rightarrow \infty$. One can produce many other interesting consequences of (1.2), such as the well known estimate for coefficients via Mahler's measure. 
Corollary 1.4. If $P_{n}(z)=\sum_{k=0}^{n} a_{k} z^{k}$ then

$$
\left|a_{k}\right| \leq\left(\begin{array}{l}
n \\
k
\end{array}\right) M\left(P_{n}\right), \quad k=1, \ldots, n .
$$

The above inequality follows at once from (1.2) by letting $\Lambda_{n}(z)=\left(\begin{array}{l}n \\ k\end{array}\right) z^{k}$, $k=1, \ldots, n$. Another interesting question is how removing a specific power term from the polynomial affects its Mahler's measure. The answer is below.

Corollary 1.5. Let $P_{n}(z)=\sum_{k=0}^{n} a_{k} z^{k}$ and $m=0, \ldots, n$. We have

$$
M\left(\sum_{k \neq m} a_{k} z^{k}\right) \leq M\left((1+z)^{n}-\left(\begin{array}{c}
n \\
m
\end{array}\right) z^{m}\right) M\left(P_{n}\right) .
$$

In particular, if $m=0$ then $M\left((1+z)^{n}-1\right)=\prod_{n / 6<k<5 n / 6} 2 \sin \frac{k \pi}{n} \approx(1.4)^{n}$ as $n \rightarrow \infty$.

Again, the proof is a simple application of $(1.2)$ with $\Lambda_{n}(z)=(1+z)^{n}-$ $\left(\begin{array}{c}n \\ m\end{array}\right) z^{m}$, so that $\lambda_{m}=0$ and $\lambda_{k}=1, k \neq m$. Finally, we state two variations of (1.2).

Corollary 1.6. If $P_{n}(z)=\sum_{k=0}^{n} a_{k} z^{k}$ and $m \in \mathbb{N}$, then

$$
M\left(\sum_{k=0}^{n} \lambda_{k}\left(\begin{array}{l}
n \\
k
\end{array}\right)^{-m} a_{k} z^{k}\right) \leq M\left(\sum_{k=0}^{n} \lambda_{k} z^{k}\right) M\left(P_{n}\right)
$$

and

$$
M\left(\sum_{k=0}^{n} \lambda_{k}^{m}\left(\begin{array}{l}
n \\
k
\end{array}\right)^{-m} a_{k} z^{k}\right) \leq M^{m}\left(\sum_{k=0}^{n} \lambda_{k} z^{k}\right) M\left(P_{n}\right)
$$

An important generalization of Theorem 1.1 for the $H^{p}$ norms was obtained by Arestov [1].

Theorem 1.7. Suppose that $P_{n} \in \mathbb{C}_{n}[z]$. If $\Lambda_{n} \in \mathbb{C}_{n}[z]$ and $\Lambda P_{n} \in \mathbb{C}_{n}[z]$ are defined by (1.1), then

$$
\left\|\Lambda P_{n}\right\|_{H^{p}} \leq M\left(\Lambda_{n}\right)\left\|P_{n}\right\|_{H^{p}}, \quad 0 \leq p \leq \infty .
$$

Recall that the Szegó composition can also be viewed as a multiplier or convolution operator in the sense of harmonic analysis. For $P_{n}(z)=\sum_{k=0}^{n} a_{k} z^{k}$, we have

$$
\Lambda P_{n}(z)=\sum_{k=0}^{n} \lambda_{k} a_{k} z^{k}=\left(\sum_{k=0}^{n} \lambda_{k} z^{k}\right) * P_{n}(z)
$$


Let $\|\Lambda\|$ be the operator norm of $\Lambda: \mathbb{C}_{n}[z] \rightarrow \mathbb{C}_{n}[z]$ :

$$
\|\Lambda\|=\sup _{P_{n} \in \mathbb{C}_{n}[z]} \frac{\left\|\Lambda P_{n}\right\|_{H^{p}}}{\left\|P_{n}\right\|_{H^{p}}}, \quad 0 \leq p \leq \infty .
$$

A particularly interesting class of multiplier operators is given by the bound (norm) preserving operators. Those are described by the condition $\left\|\Lambda P_{n}\right\|_{H^{p}} \leq$ $\left\|P_{n}\right\|_{H^{p}}$ for all $P_{n} \in \mathbb{C}_{n}[z]$, which holds if and only if $\|\Lambda\| \leq 1$. If we choose the multipliers $\lambda_{k}$ to satisfy $M\left(\Lambda_{n}\right) \leq 1$, then $\|\Lambda\| \leq 1$ by Theorem 1.7. Thus we may use the inequality $\left\|\Lambda P_{n}\right\|_{H^{p}} \leq\left\|P_{n}\right\|_{H^{p}}$ to obtain lower bounds for $\left\|P_{n}\right\|_{H^{p}}$ via a proper choice of multipliers. There are other interesting norm preserving convolution operators such as the following considered by Sheil-Small [38, pp. 168-171].

Theorem 1.8. Let $\limsup \left|\lambda_{k}\right|^{1 / k} \leq 1$. If $\lambda_{0}=1$ and

$$
\Re\left(\sum_{k=0}^{\infty} \lambda_{k} z^{k}\right)>\frac{1}{2}, \quad|z|<1,
$$

then $\left\|\Lambda P_{n}\right\|_{H^{p}} \leq\left\|P_{n}\right\|_{H^{p}}$ for any polynomial $P_{n}$, where $1 \leq p \leq \infty$.

In fact, Sheil-Small stated a result for generalized convolution operators that covers more applications. It would be interesting to explore whether Theorem 1.8 remains true for $0 \leq p<1$, i.e., for the range of $p$ including Mahler's measure.

\section{An areal analog of Mahler's measure}

A natural counterpart of Mahler's measure is obtained by replacing the normalized arclength measure on the unit circumference $\mathbb{T}$ by the normalized area measure on the unit disk $\mathbb{D}$. Namely, we define the $A^{0}$ Bergman space norm by

$$
\left\|P_{n}\right\|_{0}:=\exp \left(\frac{1}{\pi} \iint_{\mathbb{D}} \log \left|P_{n}(z)\right| d A\right) .
$$

This norm is also a multiplicative height of the polynomial $P_{n}$, cf. [32]. Furthermore, it has the same relation to Bergman spaces as Mahler's measure to Hardy spaces:

$$
\left\|P_{n}\right\|_{0}=\lim _{p \rightarrow 0+}\left\|P_{n}\right\|_{p}
$$

see [18], where

$$
\left\|P_{n}\right\|_{p}:=\left(\frac{1}{\pi} \iint_{\mathbb{D}}\left|P_{n}(z)\right|^{p} d A\right)^{1 / p}, \quad 0<p<\infty,
$$

is the $A^{p}$ Bergman space norm.

In fact, there is a direct relation between Mahler's measure and its areal analog, given below. 
Theorem 2.1. Let $P_{n}(z)=a_{n} \prod_{j=1}^{n}\left(z-z_{j}\right)=\sum_{k=0}^{n} a_{k} z^{k} \in \mathbb{C}_{n}[z]$. If $P_{n}$ has no roots in $\mathbb{D}$, then $\left\|P_{n}\right\|_{0}=M\left(P_{n}\right)=\left|a_{0}\right|$. Otherwise,

$$
\left\|P_{n}\right\|_{0}=M\left(P_{n}\right) \exp \left(\frac{1}{2} \sum_{\left|z_{j}\right|<1}\left(\left|z_{j}\right|^{2}-1\right)\right) .
$$

This shows that the value of $\left\|P_{n}\right\|_{0}$ is influenced by the zeros inside the unit disk more than that of $M\left(P_{n}\right)$. We immediately obtain the following comparison result from Theorem 2.1.

Corollary 2.2. For any $P_{n} \in \mathbb{C}_{n}[z]$, we have

$$
e^{-n / 2} M\left(P_{n}\right) \leq\left\|P_{n}\right\|_{0} \leq M\left(P_{n}\right)
$$

Equality holds in the lower estimate if and only if $P_{n}(z)=a_{n} z^{n}$. The upper estimate turns into equality for any polynomial without zeros in the unit disk.

If $P_{n}(z)=\sum_{k=0}^{n} a_{k} z^{k}$ then

$$
\left\|P_{n}\right\|_{0} \geq\left|a_{0}\right|
$$

which follows from the area mean value inequality for the subharmonic function $\log \left|P_{n}\right|$ (cf. [12]). Hence

$$
\left\|P_{n}\right\|_{0} \geq 1 \quad \text { for all } P_{n} \in \mathbb{Z}_{n}[z], P_{n}(0) \neq 0 \text {. }
$$

A well known theorem of Kronecker [21] states that any monic irreducible polynomial $P_{n} \in \mathbb{Z}_{n}[z], P_{n}(0) \neq 0$, with all zeros in the closed unit disk, must be cyclotomic. One can write that statement in the form: $M\left(P_{n}\right)=1$ for such $P_{n}$ if and only if $P_{n}$ is cyclotomic. A direct analog of this result exists for $\left\|P_{n}\right\|_{0}$.

Theorem 2.3. Suppose that $P_{n} \in \mathbb{Z}_{n}[z], P_{n}(0) \neq 0$, is an irreducible polynomial with all zeros in the closed unit disk. It is cyclotomic if and only if $\left\|P_{n}\right\|_{0}=1$.

The next natural question is whether one can find a uniform lower bound $\left\|P_{n}\right\|_{0} \geq c>1$ for all non-cyclotomic $P_{n} \in \mathbb{Z}_{n}[z], P_{n}(0) \neq 0$. It is especially interesting in view of Lehmer's conjecture, because $M\left(P_{n}\right) \geq\left\|P_{n}\right\|_{0}$ by $(2.2)$. However, the answer to the question is negative, as we show with the following example.

Example 2.4. Consider $P_{n}(z)=n z^{n}-1$. It has zeros $z_{j}, j=1, \ldots, n$, that are equally spaced on the circle $|z|=n^{-1 / n}$. Note that $M\left(P_{n}\right)=n$ and

$$
\left\|P_{n}\right\|_{0}=n \exp \left(\frac{n\left(n^{-2 / n}-1\right)}{2}\right)
$$

by (2.1). Since

$$
n^{-2 / n}=\exp \left(\frac{-2 \log n}{n}\right)=1-\frac{2 \log n}{n}+O\left(\frac{\log ^{2} n}{n^{2}}\right),
$$


we obtain that

$$
\left\|P_{n}\right\|_{0}=\exp \left(O\left(\frac{\log ^{2} n}{n}\right)\right) \rightarrow 1 \text { as } n \rightarrow \infty .
$$

Similarly, we have for the reciprocal polynomial $P_{2 n}(z)=z^{2 n}+n z^{n}+1$ that

$$
M\left(P_{n}\right)=\frac{n+\sqrt{n^{2}-4}}{2} \sim n \quad \text { as } n \rightarrow \infty,
$$

and

$\left\|P_{n}\right\|_{0}=\frac{n+\sqrt{n^{2}-4}}{2} \exp \left(\frac{n}{2}\left(\left(\frac{n+\sqrt{n^{2}-4}}{2}\right)^{2 / n}-1\right)\right) \rightarrow 1$ as $n \rightarrow \infty$.

One may notice that for both sequences of polynomials in this example the zeros are asymptotically equidistributed near the unit circle. In fact, this is a part of a more general phenomenon. Consider a polynomial $P_{n}(z)=$ $a_{n} \prod_{j=1}^{n}\left(z-z_{j}\right) \in \mathbb{C}_{n}[z]$, and define its normalized zero counting measure by

$$
\nu_{n}:=\frac{1}{n} \sum_{j=1}^{n} \delta_{z_{j}}
$$

where $\delta_{z_{j}}$ is the unit pointmass at $z_{j}$. Our main result on the asymptotic zero distribution is as follows.

Theorem 2.5. Suppose that $P_{n} \in \mathbb{Z}_{n}[z]$, $\operatorname{deg} P_{n}=n$, is a sequence of polynomials without multiple zeros. If $\lim _{n \rightarrow \infty}\left\|P_{n}\right\|_{0}^{1 / n}=1$ then the $\nu_{n}$ converge to the normalized arclength measure $d \theta /(2 \pi)$ on $\mathbb{T}$ in the weak ${ }^{*}$ topology, as $n \rightarrow \infty$.

This result extends a theorem of Bilu [4] for Mahler's measure; see also Bombieri [5] and Rumely [36]. From a more general point of view, Theorem 2.5 is a descendant of Jentzsch's result [20] on the asymptotic zero distribution of the partial sums of a power series, and its generalization by Szego" [44]. This area was further developed by Erdös and Turán [13], and by many others.

As an immediate application of Theorem 2.5 we obtain a result on the growth of $\left\|P_{n}\right\|_{0}$ for polynomials with restricted zeros.

Corollary 2.6. Suppose that $P_{n} \in \mathbb{Z}_{n}[z]$, deg $P_{n}=n$, is a sequence of polynomials with simple zeros contained in a closed set $E \subset \mathbb{C}$. If $\mathbb{T} \not \subset E$ then there exists a constant $C=C(E)>1$ such that

$$
\liminf _{n \rightarrow \infty}\left\|P_{n}\right\|_{0}^{1 / n} \geq C>1
$$

This exhibits the geometric growth of $\left\|P_{n}\right\|_{0}$ for many families of polynomials such as polynomials with real zeros, polynomials with zeros in a sector, etc. Corresponding results with explicit bounds for Mahler's measure were obtained by Schinzel [37], Langevin [22, 23, 24], Mignotte [30], Rhin and 
Smyth [35], Dubickas and Smyth [11], and others. A detailed account of these results is contained in Smyth [41].

In a somewhat different direction, we have the following result on the asymptotic behavior of zeros.

Theorem 2.7. Suppose that $P_{n}(z)=a_{n} z^{n}+\ldots+a_{0} \in \mathbb{C}_{n}[z],\left|a_{0}\right| \geq 1, n \in \mathbb{N}$, is a sequence of polynomials.

(a) If $\lim _{n \rightarrow \infty}\left\|P_{n}\right\|_{0}=1$ then

$$
\liminf _{n \rightarrow \infty} \min _{1 \leq j \leq n}\left|z_{j}\right| \geq 1 \text {. }
$$

(b) If $\left|a_{n}\right| \geq 1$ and $\lim _{n \rightarrow \infty} M\left(P_{n}\right)=1$, then

$$
\lim _{n \rightarrow \infty} \min _{1 \leq j \leq n}\left|z_{j}\right|=\lim _{n \rightarrow \infty} \max _{1 \leq j \leq n}\left|z_{j}\right|=1 .
$$

Thus part (a) of Theorem 2.7 indicates that all zeros of $P_{n}$ are pushed out of $\mathbb{D}$ as $n \rightarrow \infty$, while in part (b) they all tend to the unit circle.

\section{Polynomial inequalities in Bergman spaces}

We obtain the following generalization of Theorems 1.1 and 1.7 for the Bergman space norms.

Theorem 3.1. Suppose that $P_{n} \in \mathbb{C}_{n}[z]$. If $\Lambda_{n} \in \mathbb{C}_{n}[z]$ and $\Lambda P_{n} \in \mathbb{C}_{n}[z]$ are defined by (1.1), then

$$
\left\|\Lambda P_{n}\right\|_{p} \leq M\left(\Lambda_{n}\right)\left\|P_{n}\right\|_{p}, \quad 0 \leq p \leq \infty .
$$

Note that equality holds in (3.1) for any polynomial $P_{n} \in \mathbb{C}_{n}[z]$ when $\Lambda_{n}(z)=(1+z)^{n}=\sum_{k=0}^{n}\left(\begin{array}{l}n \\ k\end{array}\right) z^{k}$, because $\Lambda P_{n} \equiv P_{n}$ and $M\left((1+z)^{n}\right)=1$. This inequality allows to treat many problems in a unified way, and it has numerous interesting consequences. Theorem 3.1 implies that $z^{n}$ has the smallest Bergman space norm among all monic polynomials.

Corollary 3.2. If $P_{n} \in \mathbb{C}_{n}[z]$ is a monic polynomial, then

$$
\left\|P_{n}\right\|_{p} \geq\left\|z^{n}\right\|_{p}= \begin{cases}e^{-n / 2}, & p=0, \\ \left(\frac{2}{p n+2}\right)^{1 / p}, & 0<p<\infty .\end{cases}
$$

It is well known that $\left\|P_{n}\right\|_{\infty} \geq\left\|z^{n}\right\|_{\infty}=1$, see [7, 33].

Another useful estimate compares norms on the concentric disks $D_{R}:=$ $\{z:|z|<R\}$ to that on the unit disk.

Corollary 3.3. If $P_{n} \in \mathbb{C}_{n}[z]$ and $R \geq 1$, then

$$
\left(\frac{1}{\pi R^{2}} \iint_{D_{R}}\left|P_{n}(z)\right|^{p} d A\right)^{1 / p} \leq R^{n}\left\|P_{n}\right\|_{p}, \quad p \in(0, \infty),
$$


and

$$
\exp \left(\frac{1}{\pi R^{2}} \iint_{D_{R}} \log \left|P_{n}(z)\right| d A\right) \leq R^{n}\left\|P_{n}\right\|_{0}
$$

where equality holds for $P_{n}(z)=z^{n}$.

Again, in the case $p=\infty$, it is already known that $\max _{z \in D_{R}}\left|P_{n}(z)\right| \leq$ $R^{n}\left\|P_{n}\right\|_{\infty}$. See [33].

Another consequence relates $\left\|P_{n}\right\|_{p}$ to the coefficients of $P_{n}$.

Corollary 3.4. If $P_{n}(z)=\sum_{k=0}^{n} a_{k} z^{k} \in \mathbb{C}_{n}[z]$ then

$$
\left|a_{k}\right| \leq\left(\frac{p k+2}{2}\right)^{1 / p}\left(\begin{array}{l}
n \\
k
\end{array}\right)\left\|P_{n}\right\|_{p}, \quad k=0, \ldots, n, 0<p<\infty,
$$

and

$$
\left|a_{k}\right| \leq e^{k / 2}\left(\begin{array}{l}
n \\
k
\end{array}\right)\left\|P_{n}\right\|_{0}, \quad k=0, \ldots, n .
$$

One can certainly extend the list of corollaries by choosing appropriate polynomials $\Lambda_{n}$.

3.1. Bernstein-type inequalities. The original Bernstein inequality (cf. [7], [31] and [33]) gives an estimate for the supremum norm of the derivative of a polynomial on $D$ :

$$
\left\|P_{n}^{\prime}\right\|_{\infty} \leq n\left\|P_{n}\right\|_{\infty}, \quad P_{n} \in \mathbb{C}_{n}[z] .
$$

Its sharpness is easily seen by considering $P_{n}(z)=z^{n}$. Zygmund [47] extended this result to the Hardy spaces by proving that

$$
\left\|P_{n}^{\prime}\right\|_{H^{p}} \leq n\left\|P_{n}\right\|_{H^{p}}, \quad p \in[1, \infty) .
$$

As we know from the first section, De Bruijn and Springer [10], and later Mahler [26], showed that

$$
M\left(P_{n}^{\prime}\right) \leq n M\left(P_{n}\right),
$$

thus settling the case $p=0$ for the Hardy space norms. It had been an open question for a long time, whether the above inequality is true for $0<p<1$. After a partial result of Máté and Nevai [29], the question was answered in the affirmative by Arestov [1].

We obtain the following version of the Bernstein inequality for Bergman spaces, as a consequence of Theorem 3.1.

Theorem 3.5. For any $P_{n} \in \mathbb{C}_{n}[z]$, we have that

$$
\left\|z P_{n}^{\prime}\right\|_{p} \leq n\left\|P_{n}\right\|_{p}, \quad 0 \leq p<\infty .
$$

Note that equality holds here for $P_{n}(z)=z^{n}$.

It is also of interest to find the Bernstein inequalities in Bergman spaces exactly matching the classical one in form. For example, when $p=0$, we have 
Corollary 3.6. If $P_{n} \in \mathbb{C}_{n}[z]$ then

$$
\left\|P_{n}^{\prime}\right\|_{0} \leq \sqrt{e} n\left\|P_{n}\right\|_{0},
$$

where equality holds for $P_{n}(z)=z^{n}$.

Furthermore, we obtain by an elementary argument the following

Proposition 3.7. If $P_{n} \in \mathbb{C}_{n}[z]$ then

$$
\left\|P_{n}^{\prime}\right\|_{2} \leq \sqrt{n(n+1)}\left\|P_{n}\right\|_{2}
$$

with equality for $P_{n}(z)=z^{n}$.

This suggests that, for arbitrary $p \in(0, \infty)$, one might be able to prove

Conjecture 3.8. If $P_{n} \in \mathbb{C}_{n}[z]$ then

$$
\left\|P_{n}^{\prime}\right\|_{p} \leq n\left(1+\frac{1}{n-1+2 / p}\right)^{1 / p}\left\|P_{n}\right\|_{p}, \quad 0<p<\infty,
$$

with equality for $P_{n}(z)=z^{n}$.

Note that Corollary 3.6 may be viewed as the limiting case of this conjecture as $p \rightarrow 0$, while the classical Bernstein inequality is obtained by letting $p \rightarrow$ $\infty$.

3.2. Comparing the Hardy and the Bergman norms. It is well known $[12,19]$ that for any function $f \in H^{p}$ we have

$$
\|f\|_{p} \leq\|f\|_{H^{p}}, \quad 0 \leq p \leq \infty .
$$

Clearly, we have equality for $p=\infty$. One can prove inequalities for polynomials in the opposite direction, of the form

$$
\left\|P_{n}\right\|_{H^{p}} \leq C(n, p)\left\|P_{n}\right\|_{p}
$$

For example, we have for $p=0$ that

$$
M\left(P_{n}\right) \leq e^{n / 2}\left\|P_{n}\right\|_{0},
$$

where equality holds for $P_{n}(z)=z^{n}$ (see Corollary 2.2).

The case $p=2$ is easy to handle, because

$$
\left\|P_{n}\right\|_{H^{2}}^{2}=\sum_{k=0}^{n}\left|a_{k}\right|^{2} \leq(n+1) \sum_{k=0}^{n} \frac{\left|a_{k}\right|^{2}}{k+1}=(n+1)\left\|P_{n}\right\|_{2}^{2},
$$

where $P_{n}(z)=\sum_{k=0}^{n} a_{k} z^{k}$. Hence

Proposition 3.9. If $P_{n} \in \mathbb{C}_{n}[z]$ then

$$
\left\|P_{n}\right\|_{H^{2}} \leq \sqrt{n+1}\left\|P_{n}\right\|_{2}
$$

with equality for $P_{n}(z)=z^{n}$.

It is likely that the following is true. 
Conjecture 3.10. If $P_{n} \in \mathbb{C}_{n}[z]$ then

$$
\left\|P_{n}\right\|_{H^{p}} \leq(p n / 2+1)^{1 / p}\left\|P_{n}\right\|_{p}, \quad 0<p<\infty,
$$

with equality for $P_{n}(z)=z^{n}$.

This holds in the limit for $p=\infty$ (trivially) and for $p=0$ by (3.11).

\section{Approximation by polynomials With integer COEFFicients}

We consider a related question of approximation by polynomials with integer coefficients on the unit disk. There is a well known condition necessary for approximation by integer polynomials in essentially any norm on $\mathbb{D}$.

Proposition 4.1. Suppose that $P_{n} \in \mathbb{Z}_{n}[z], n \in \mathbb{N}$, converge to $f$ uniformly on compact subsets of $\mathbb{D}$. Then $f$ is analytic in $\mathbb{D}$ and $f^{(k)}(0) / k ! \in \mathbb{Z}$ for all $k \geq 0, k \in \mathbb{Z}$.

This necessary condition for the convergence is clearly equivalent to the fact that the power series expansion of $f$ at the origin has integer coefficients.

It is well known that approximation by polynomials with integer coefficients is possible in $H^{p}$ only in the trivial case. See [16] and [45]. More precisely, we have

Proposition 4.2. Suppose that $f \in H^{p}, 0<p \leq \infty$. If $P_{n} \in \mathbb{Z}_{n}[z], n \in \mathbb{N}$, satisfy

$$
\lim _{n \rightarrow \infty}\left\|f-P_{n}\right\|_{H^{p}}=0,
$$

then $f$ is a polynomial with integer coefficients.

It remains an open question whether this proposition is true for $p=0$, i.e. for approximation of functions in Mahler's measure. One can see from the proof of Proposition 4.2, given in Section 6.4, that the main obstacle is that we have no substitute for the triangle inequality in the case of Mahler's measure. Mahler [27] raised an interesting question related to this problem. While it is not possible to have $M(f+g) \leq C(M(f)+M(g))$ for a fixed constant $C$, in general, one can consider a natural analog of the triangle inequality for all polynomials $P_{n}, Q_{n} \in \mathbb{C}_{n}[z]$ :

$$
M\left(P_{n}+Q_{n}\right) \leq c^{n}\left(M\left(P_{n}\right)+M\left(Q_{n}\right)\right) .
$$

Mahler [27] showed that $c=2$ is possible in the above inequality, and asked what is the best (smallest) value of $c$. He later improved the constant $c$ in [28], and the best currently known range $1.7916<c<1.8493$ was obtained by Arestov [2].

Generally, nontrivial approximation by integer polynomials in the supremum norm is valid on sets with transfinite diameter (capacity) less than 1 $[16,45]$, and it is not possible if the transfinite diameter is greater than or equal to 1 . But the transfinite diameter of $\mathbb{D}$ is exactly equal to 1 , so that we deal with a borderline case. However, we show that the Bergman space $A^{p}$ 
is different from the Hardy space $H^{p}$ in this regard, as it does allow approximation by polynomials with integer coefficients.

Theorem 4.3. Suppose that $f \in A^{p}, 1<p<\infty$. We have

$$
\lim _{n \rightarrow \infty}\left\|f-P_{n}\right\|_{p}=0
$$

for a sequence of polynomials $P_{n} \in \mathbb{Z}_{n}[z], n \in \mathbb{N}$, if and only if $f$ has a power series expansion about $z=0$ with integer coefficients. Clearly, this is equivalent to $f^{(k)}(0) / k ! \in \mathbb{Z}$ for all $k \geq 0, k \in \mathbb{Z}$.

Thus there are many functions in $A^{p}$ that can be approximated by polynomials with integer coefficients. In fact, one can use partial sums of the power series for this purpose. See the proof of Theorem 4.3. However, we do not know whether Theorem 4.3 is valid in the case $0 \leq p \leq 1$. Note that if $f \in A^{p}, p>1$, has a Taylor expansion with integer coefficients, then $f \in A^{q}$ for any $q \in[0, p)$ and the partial sums $P_{n}$ of this expansion satisfy $\left\|f-P_{n}\right\|_{q} \leq\left\|f-P_{n}\right\|_{p} \rightarrow 0$ as $n \rightarrow \infty$.

\section{Multivariate POLYNOMials}

We believe that many of the results mentioned in this survey are capable of generalizations to the multivariate case. However, we do not try to accomplish such an ambitious program here, and restrict ourselves to a few simple remarks. The definition of $\left\|P_{n}\right\|_{0}$ is easily generalized to the case of multivariate polynomials $P_{n}\left(z_{1}, \ldots, z_{d}\right)$ as follows:

$$
\left\|P_{n}\right\|_{0}:=\exp \left(\frac{1}{\pi^{d}} \int_{\mathbb{D}} \ldots \int_{\mathbb{D}} \log \left|P_{n}\left(z_{1}, \ldots, z_{d}\right)\right| d A\left(z_{1}\right) \ldots d A\left(z_{d}\right)\right) .
$$

It is also parallel to multivariate Mahler's measure

$$
M\left(P_{n}\right):=\exp \left(\frac{1}{(2 \pi)^{d}} \int_{\mathbb{T}} \ldots \int_{\mathbb{T}} \log \left|P_{n}\left(z_{1}, \ldots, z_{d}\right)\right|\left|d z_{1}\right| \ldots\left|d z_{d}\right|\right) .
$$

We note that many of the properties of $\left\|P_{n}\right\|_{0}$ are preserved in the multivariate case. Thus it still defines a multiplicative height on the space of polynomials. If $P_{n}$ is a polynomial with complex coefficients and the constant term $a_{0}$, then we can apply the area mean value inequality to the (pluri)subharmonic function $\log \left|P_{n}\left(z_{1}, \ldots, z_{d}\right)\right|$ in each variable, which gives together with Fubini's theorem that

$$
\left\|P_{n}\right\|_{0} \geq\left|a_{0}\right|
$$

Furthermore, the above inequality turns into equality if $P_{n}\left(z_{1}, \ldots, z_{d}\right) \neq 0$ on $\mathbb{D}^{d}$, by the area mean value theorem for the (pluri)harmonic function $\log \left|P_{n}\left(z_{1}, \ldots, z_{d}\right)\right|$. However, it is rather unlikely that some kind of explicit relation such as (2.1) exists for general multivariate polynomials.

We now state an estimate generalizing Corollary 2.2. 
Proposition 5.1. For a polynomial

$$
P_{n}\left(z_{1}, \ldots, z_{d}\right)=\sum_{k_{1}+\ldots+k_{d} \leq n} a_{k_{1} \ldots k_{d}} z_{1}^{k_{1}} \ldots z_{d}^{k_{d}}
$$

of degree at most $n$ with complex coefficients, we have

$$
e^{-n / 2} M\left(P_{n}\right) \leq\left\|P_{n}\right\|_{0} \leq M\left(P_{n}\right) .
$$

Equality holds in the lower estimate for any $P_{n}\left(z_{1}, \ldots, z_{d}\right)=a_{k_{1} \ldots k_{d}} z_{1}^{k_{1}} \ldots z_{d}^{k_{d}}$ with $k_{1}+\ldots+k_{d}=n$. The upper estimate turns into equality for any polynomial not vanishing in $\mathbb{D}^{d}$.

It is of interest to find explicit values of the multivariate $\left\|P_{n}\right\|_{0}$. This problem has received a considerable attention in Mahler's measure setting (see [9], $[39,40],[14],[17])$, and it remains a very active area of research. In particular, it is of importance to characterize multivariate polynomials with integer coefficients satisfying $\left\|P_{n}\right\|_{0}=1$. Smyth [40] proved a complete Kronecker-type characterization for the multivariate Mahler's measure $M\left(P_{n}\right)=1$. Thus we expect that one should be able to produce an analog for $\left\|P_{n}\right\|_{0}$, generalizing Theorem 2.3.

Example 5.2. The following identities hold for the multivariate $\left\|P_{n}\right\|_{0}$ :

(a) $\left\|z_{1}+z_{2}\right\|_{0}=e^{-1 / 4}$

(b) $\left\|1+z_{1}^{k_{1}} \ldots z_{d}^{k_{d}}\right\|_{0}=1, \quad k_{1}, \ldots, k_{d} \geq 0$

(c) If the polynomial $P_{n}$ of the form (5.1) satisfies

$$
\left|a_{0 \ldots 0}\right| \geq \sum_{0<k_{1}+\ldots+k_{d} \leq n}\left|a_{k_{1} \ldots k_{d}}\right|
$$

then $\left\|P_{n}\right\|_{0}=M\left(P_{n}\right)=\left|a_{0 \ldots 0}\right|$.

\section{ProOFs}

\subsection{Proofs for Section 1.}

Proof of Corollary 1.6. Let $\lambda_{k} \in \mathbb{C}, k=0, \ldots, n$, be arbitrary fixed numbers, and define the operator

$$
A_{m} P_{n}(z):=\sum_{k=0}^{n} \lambda_{k}\left(\begin{array}{l}
n \\
k
\end{array}\right)^{-m} a_{k} z^{k}
$$

It is clear from (1.2) that

$$
M\left(A_{1} P_{n}\right)=M\left(\sum_{k=0}^{n} \lambda_{k}\left(\begin{array}{l}
n \\
k
\end{array}\right)^{-1} a_{k} z^{k}\right) \leq M\left(\sum_{k=0}^{n} \lambda_{k} z^{k}\right) M\left(P_{n}\right),
$$

which we use as the basis of induction in $m$. Assuming that

$$
M\left(A_{m} P_{n}\right) \leq M\left(\sum_{k=0}^{n} \lambda_{k} z^{k}\right) M\left(P_{n}\right)
$$


holds, we obtain that

$$
\begin{aligned}
M\left(A_{m+1} P_{n}\right) & =M\left(\sum_{k=0}^{n} \lambda_{k}\left(\begin{array}{l}
n \\
k
\end{array}\right)^{-m-1} a_{k} z^{k}\right) \\
& \leq M\left(\sum_{k=0}^{n} \lambda_{k}\left(\begin{array}{l}
n \\
k
\end{array}\right)^{-m} a_{k} z^{k}\right) M\left(\sum_{k=0}^{n} z^{k}\right),
\end{aligned}
$$

where we used (6.1) with $\lambda_{k}$ replaced by $\lambda_{k}\left(\begin{array}{l}n \\ k\end{array}\right)^{-m} a_{k}$, and with $P_{n}(z)=\sum_{k=0}^{n} z^{k}$. Since $M\left(\sum_{k=0}^{n} z^{k}\right)=1$, it follows that

$$
M\left(A_{m+1} P_{n}\right) \leq M\left(A_{m} P_{n}\right), \quad m \in \mathbb{N},
$$

and (1.3) is proved by the induction hypothesis.

Let

$$
B P_{n}(z):=\sum_{k=0}^{n} \lambda_{k}\left(\begin{array}{l}
n \\
k
\end{array}\right)^{-1} a_{k} z^{k}=A_{1} P_{n}(z),
$$

so that its $m$-fold composition is

$$
B^{m} P_{n}(z)=\sum_{k=0}^{n} \lambda_{k}^{m}\left(\begin{array}{l}
n \\
k
\end{array}\right)^{-m} a_{k} z^{k}
$$

Applying (6.1) $m$ times, we arrive at (1.4).

\subsection{Proofs for Section 2.}

Proof of Theorem 2.1. If $P_{n}$ does not vanish in $\mathbb{D}$, then $\log \left|P_{n}(z)\right|$ is harmonic in $\mathbb{D}$. Hence $M\left(P_{n}\right)=\left|a_{0}\right|$ and $\left\|P_{n}\right\|_{0}=\left|a_{0}\right|$ follow from the contour and area mean value theorems. Assume now that $P_{n}$ has zeros in $\mathbb{D}$. Applying Jensen's formula, we obtain that

$$
\log M\left(P_{n}\right)=\frac{1}{2 \pi} \int_{0}^{2 \pi} \log \left|P_{n}\left(e^{i \theta}\right)\right| d \theta=\log \left|a_{n}\right|+\sum_{\left|z_{j}\right| \geq 1} \log \left|z_{j}\right| .
$$

Furthermore,

$$
\begin{aligned}
\log \left\|P_{n}\right\|_{0} & =\frac{1}{\pi} \int_{0}^{1} \int_{0}^{2 \pi} \log \left|P_{n}\left(r e^{i \theta}\right)\right| r d r d \theta \\
& =2 \int_{0}^{1}\left(\frac{1}{2 \pi} \int_{0}^{2 \pi} \log \left|P_{n}\left(r e^{i \theta}\right)\right| d \theta\right) r d r \\
& =2 \int_{0}^{1}\left(\log \left|a_{n}\right|+\sum_{\left|z_{j}\right| \geq r} \log \left|z_{j}\right|+\sum_{\left|z_{j}\right|<r} \log r\right) r d r \\
& =\log \left|a_{n}\right|+\sum_{\left|z_{j}\right| \geq 1} \log \left|z_{j}\right|+\frac{1}{2} \sum_{\left|z_{j}\right|<1}\left(\left|z_{j}\right|^{2}-1\right) .
\end{aligned}
$$


Hence

$$
\left\|P_{n}\right\|_{0}=M\left(P_{n}\right) \exp \left(\frac{1}{2} \sum_{\left|z_{j}\right|<1}\left(\left|z_{j}\right|^{2}-1\right)\right) \text {. }
$$

Proof of Corollary 2.2. Inequality (2.2) follows from (2.1) after observing that the smallest value of the exponential is achieved when all $z_{j}=0$, while the largest value is 1 when all $\left|z_{j}\right| \geq 1$.

Proof of Theorem 2.3. If $P_{n}$ is cyclotomic, then $\left\|P_{n}\right\|_{0}=1$ by Theorem 2.1, because $\left|z_{j}\right|=1, j=1, \ldots, n$, and $M\left(P_{n}\right)=1$. Assume now that $\left\|P_{n}\right\|_{0}=1$. Let $z_{j}, j=1, \ldots, m, m \leq n$, be the zeros of $P_{n}$ in $\mathbb{D}$. Then we have from (2.1) that

$$
\left\|P_{n}\right\|_{0}=\left|a_{0}\right| \prod_{j=1}^{m} \frac{e^{\left(\left|z_{j}\right|^{2}-1\right) / 2}}{\left|z_{j}\right|} \geq \prod_{j=1}^{m} \frac{e^{\left(\left|z_{j}\right|^{2}-1\right) / 2}}{\left|z_{j}\right|}
$$

where $a_{0} \neq 0$ is the constant term of $P_{n}$. Define $g(x):=e^{\left(x^{2}-1\right) / 2} / x, x>0$, and observe that $g^{\prime}(x)<0$ when $x \in(0,1)$, while $g^{\prime}(x)>0$ when $x \in(1, \infty)$. Hence

$$
g(1)=1 \text { is the strict global minimum for } g(x) \text { on }(0, \infty) \text {. }
$$

It follows from (6.2)-(6.3) that

$$
1<\prod_{j=1}^{m} g\left(\left|z_{j}\right|\right)=\prod_{j=1}^{m} \frac{e^{\left(\left|z_{j}\right|^{2}-1\right) / 2}}{\left|z_{j}\right|} \leq\left\|P_{n}\right\|_{0}=1
$$

which is a contradiction. Hence $P_{n}$ has no zeros in $\mathbb{D}$, and $M\left(P_{n}\right)=\left\|P_{n}\right\|_{0}=1$ by Theorem 2.1. This implies that $P_{n}$ is cyclotomic by Kronecker's theorem.

We could also proceed in a different way, by assuming that $\left\|P_{n}\right\|_{0}=1$ and observing from (6.2) that

$$
\exp \left(\sum_{j=1}^{m} \frac{\left|z_{j}\right|^{2}-1}{2}\right)=\frac{1}{\left|a_{0}\right|} \prod_{j=1}^{m}\left|z_{j}\right|
$$

Since the expression on the right is an algebraic number, as well as the sum in the exponent on the left, we obtain that equality is only possible when the latter sum is zero, by the well known result of Lindemann that the exponential of a nonzero algebraic number is transcendental [3]. Hence $\left|z_{j}\right| \geq 1, j=$ $1, \ldots, n$, and $M\left(P_{n}\right)=\left\|P_{n}\right\|_{0}=1$ as before.

Proof of Theorem 2.5. We first show that $P_{n}$ has $o(n)$ zeros in $D_{r}:=\{z$ : $|z|<r\}$ as $n \rightarrow \infty$, for any $r<1$. Assume to the contrary that there is a subsequence of $n$ such that $P_{n}$ has at least $\alpha n$ zeros, with $\alpha>0$, in some 
$D_{r}, r<1$. Suppose that those zeros are $z_{j} \neq 0, j=1, \ldots, m, m \leq n$, and proceed as in the proof of Theorem 2.3 to obtain

$$
\prod_{j=1}^{m} g\left(\left|z_{j}\right|\right)=\prod_{j=1}^{m} \frac{e^{\left(\left|z_{j}\right|^{2}-1\right) / 2}}{\left|z_{j}\right|} \leq\left\|P_{n}\right\|_{0}
$$

by (6.2). Since $g(x)=e^{\left(x^{2}-1\right) / 2} / x$ is strictly decreasing on $(0,1)$, we have that

$$
\prod_{j=1}^{m} g\left(\left|z_{j}\right|\right) \geq(g(r))^{\alpha n}
$$

It immediately follows from (6.3) and (6.4) that

$$
\limsup _{n \rightarrow \infty}\left\|P_{n}\right\|_{0}^{1 / n} \geq(g(r))^{\alpha}>1
$$

which is in direct conflict with assumptions of this theorem. If $P_{n}$ has a simple zero at $z=0$, then $P_{n}(z)=z Q_{n-1}(z)$ and $\left\|P_{n}\right\|_{0}=\left\|Q_{n-1}\right\|_{0} / \sqrt{e}$. Hence we can apply the above argument to $Q_{n-1}$ and come to the same conclusion that $P_{n}$ has $o(n)$ zeros in $D_{r}:=\{z:|z|<r\}, r<1$, as $n \rightarrow \infty$.

The second step is to show that $\lim _{n \rightarrow \infty}\left(M\left(P_{n}\right)\right)^{1 / n}=1$. Note that

$$
1 \leq M\left(P_{n}\right)=\left\|P_{n}\right\|_{0} \exp \left(\frac{1}{2} \sum_{\left|z_{j}\right|<1}\left(1-\left|z_{j}\right|^{2}\right)\right) .
$$

If $P_{n}$ has $m=o(n)$ zeros in $D_{r}, r<1$, then

$$
\exp \left(\frac{1}{2} \sum_{\left|z_{j}\right|<1}\left(1-\left|z_{j}\right|^{2}\right)\right) \leq e^{m / 2+n\left(1-r^{2}\right) / 2} .
$$

Using this in (6.5), we obtain that

$$
\begin{aligned}
1 & \leq \liminf _{n \rightarrow \infty}\left(M\left(P_{n}\right)\right)^{1 / n} \leq \limsup _{n \rightarrow \infty}\left(M\left(P_{n}\right)\right)^{1 / n} \\
& \leq e^{\left(1-r^{2}\right) / 2} \lim _{n \rightarrow \infty}\left\|P_{n}\right\|_{0}^{1 / n}=e^{\left(1-r^{2}\right) / 2} .
\end{aligned}
$$

Hence $\lim _{n \rightarrow \infty}\left(M\left(P_{n}\right)\right)^{1 / n}=1$ follows by letting $r \rightarrow 1-$. The proof may now be completed by applying Bilu's result [4] (at least when $P_{n}$ is irreducible for all $n \in \mathbb{N}$ ), but we prefer to continue with an independent proof via a standard potential theoretic argument.

Observe that $P_{n}(z)=a_{n} \prod_{j=1}^{n}\left(z-z_{j}\right)$ has $o(n)$ zeros in $\mathbb{C} \backslash D_{r}, r>1$, for otherwise we would have $\liminf _{n \rightarrow \infty}\left(M\left(P_{n}\right)\right)^{1 / n}>1$ as

$$
M\left(P_{n}\right)=\left|a_{n}\right| \prod_{\left|z_{j}\right|>1}\left|z_{j}\right| \geq \prod_{\left|z_{j}\right|>1}\left|z_{j}\right| .
$$

This also implies that

$$
\lim _{n \rightarrow \infty}\left|a_{n}\right|^{1 / n}=1 .
$$


Hence any weak* limit $\nu$ of the sequence $\nu_{n}$ must satisfy $\operatorname{supp} \nu \subset \mathbb{T}$. Define the logarithmic energy of $\nu$ by

$$
I(\nu):=\iint \log \frac{1}{|z-t|} d \nu(z) d \nu(t) .
$$

Our goal is to show that $I(\nu)=0$, which implies that $\nu$ has the smallest possible energy among all positive Borel measures of mass 1 supported on $\mathbb{T}$. On the other hand, it is well known in potential theory that the equilibrium measure minimizing the energy integral is unique, and it is equal to the normalized arclength on $\mathbb{T}[34,46]$. Thus $\nu=d \theta /(2 \pi)$ and the proof would be completed.

Define the discriminant of $P_{n}$ as $\Delta_{n}:=a_{n}^{2 n-2} \prod_{1 \leq j<k \leq n}\left(z_{j}-z_{k}\right)^{2}$. Observe that it is an integer, being a symmetric form with integer coefficients in the roots of $P_{n} \in \mathbb{Z}_{n}[z]$. Since $P_{n}$ has no multiple roots, we have $\Delta_{n} \neq 0$ and $\left|\Delta_{n}\right| \geq 1$. Therefore

$$
\log \frac{1}{\left|\Delta_{n}\right|}=-(2 n-2) \log \left|a_{n}\right|+\sum_{j \neq k} \log \frac{1}{\left|z_{j}-z_{k}\right|} \leq 0 .
$$

Let

$$
K_{M}(z, t):=\min \left(\log \frac{1}{|z-t|}, M\right), \quad M>0 .
$$

It is clear that $K_{M}(z, t)$ is a continuous function in $z$ and $t$ on $\mathbb{C} \times \mathbb{C}$, and that $K_{M}(z, t)$ increases to $\log \frac{1}{|z-t|}$ as $M \rightarrow \infty$. Using the Monotone Convergence Theorem and the weak ${ }^{*}$ convergence of $\nu_{n} \times \nu_{n}$ to $\nu \times \nu$, we obtain that

$$
\begin{aligned}
I(\nu) & =\lim _{M \rightarrow \infty} \iint K_{M}(z, t) d \nu(z) d \nu(t) \\
& =\lim _{M \rightarrow \infty}\left(\lim _{n \rightarrow \infty} \iint K_{M}(z, t) d \nu_{n}(z) d \nu_{n}(t)\right) \\
& =\lim _{M \rightarrow \infty}\left(\lim _{n \rightarrow \infty}\left(\frac{1}{n^{2}} \sum_{j \neq k} K_{M}\left(z_{j}, z_{k}\right)+\frac{M}{n}\right)\right) \\
& \leq \lim _{M \rightarrow \infty}\left(\liminf _{n \rightarrow \infty} \frac{1}{n^{2}} \sum_{j \neq k} \log \frac{1}{\left|z_{j}-z_{k}\right|}\right) \\
& =\liminf _{n \rightarrow \infty} \frac{1}{n^{2}} \log \frac{\left|a_{n}\right|^{2 n-2}}{\Delta_{n}} .
\end{aligned}
$$

Hence $I(\nu) \leq 0$ follows from (6.6)-(6.7). But $I(\mu)>0$ for any positive unit Borel measure supported on $\mathbb{T}$, with the only exception for the equilibrium measure $d \mu_{\mathbb{T}}:=d \theta /(2 \pi), I\left(\mu_{\mathbb{T}}\right)=0$, see [46, pp. 53-89].

Proof of Theorem 2.7. (a) We use the same notation and approach as in the proof of Theorem 2.3. If $P_{n}$ has no zeros in $\mathbb{D}$, then $\min _{1 \leq j \leq n}\left|z_{j}\right| \geq 1$. 
POLYNOMIAL INEQUALITIES, MAHLER'S MEASURE, AND MULTIPLIERS 271

Otherwise, let $z_{j}, j=1, \ldots, m, m \leq n$, be the zeros of $P_{n}$ in $\mathbb{D}$. It follows from (6.2)-(6.3) that

$$
\left\|P_{n}\right\|_{0}=\left|a_{0}\right| \prod_{j=1}^{m} \frac{e^{\left(\left|z_{j}\right|^{2}-1\right) / 2}}{\left|z_{j}\right|} \geq g\left(\min _{1 \leq j \leq n}\left|z_{j}\right|\right)>1 .
$$

Thus we obtain the result by the continuity of $g(x)=e^{\left(x^{2}-1\right) / 2} / x, x>0$, and (6.3).

(b) Note that $\lim _{n \rightarrow \infty}\left\|P_{n}\right\|_{0}=1$ in this case too, by (2.2) and (2.3). Hence (2.5) holds true. Furthermore, we have for any zero $z_{k} \in \mathbb{C} \backslash \mathbb{D}$ that

$$
1 \leq\left|z_{k}\right| \leq\left|a_{n}\right| \prod_{\left|z_{j}\right|>1}\left|z_{j}\right|=M\left(P_{n}\right)
$$

Thus

$$
\lim _{n \rightarrow \infty} \max _{1 \leq j \leq n}\left|z_{j}\right|=1
$$

and (2.6) follows.

\subsection{Proofs for Section 3.}

Proof of Theorem 3.1. Using (1.2) for the polynomial $P_{n}(r z), r \in[0,1]$, we obtain that

$$
\frac{1}{2 \pi} \int_{0}^{2 \pi} \log \left|\Lambda P_{n}\left(r e^{i \theta}\right)\right| d \theta \leq \log M\left(\Lambda_{n}\right)+\frac{1}{2 \pi} \int_{0}^{2 \pi} \log \left|P_{n}\left(r e^{i \theta}\right)\right| d \theta .
$$

Hence (3.1) follows for $p=0$, if we multiply this inequality by $r d r$ and integrate from 0 to 1 . Similarly, we obtain from (1.5) that

$$
\frac{1}{2 \pi} \int_{0}^{2 \pi}\left|\Lambda P_{n}\left(r e^{i \theta}\right)\right|^{p} d \theta \leq \frac{M^{p}\left(\Lambda_{n}\right)}{2 \pi} \int_{0}^{2 \pi}\left|P_{n}\left(r e^{i \theta}\right)\right|^{p} d \theta, \quad 0<p<\infty,
$$

which gives (3.1) for this range of $p$ after integration with respect to $r d r$. When $p=\infty,(3.1)$ is identical to (1.5).

Proof of Corollary 3.2. Consider a monic polynomial $P_{n}(z)=z^{n}+\ldots$ and $\Lambda_{n}(z)=z^{n}$. Then $\Lambda P_{n}(z)=z^{n}$, so that (3.2) follows from (3.1) and an elementary computation.

Proof of Corollary 3.3. Let $\Lambda_{n}(z)=(1+R z)^{n}=\sum_{k=0}^{n}\left(\begin{array}{l}n \\ k\end{array}\right) R^{k} z^{k}$. Then $\Lambda P_{n}(z)=P_{n}(R z)$ and $M\left(\Lambda_{n}\right)=R^{n}$. Hence (3.1) gives that

$$
\left\|P_{n}(R z)\right\|_{p} \leq R^{n}\left\|P_{n}\right\|_{p}, \quad 0 \leq p<\infty \text { and } R \geq 1 .
$$

Changing variable and passing to the integrals over $D_{R}$, we obtain (3.3) and (3.4). The case of equality for $P_{n}(z)=z^{n}$ is verified by a routine calculation. 
Proof of Corollary 3.4. Let $\Lambda_{n}(z)=\left(\begin{array}{l}n \\ k\end{array}\right) z^{k}, 0 \leq k \leq n$. Then $\Lambda P_{n}(z)=a_{k} z^{k}$ and $M\left(\Lambda_{n}\right)=\left(\begin{array}{l}n \\ k\end{array}\right)$. It follows from (3.1) that

$$
\left|a_{k}\right|\left\|z^{k}\right\|_{p}=\left\|a_{k} z^{k}\right\|_{p} \leq\left(\begin{array}{l}
n \\
k
\end{array}\right)\left\|P_{n}\right\|_{p}, \quad 0 \leq p \leq \infty .
$$

One only needs now to find $\left\|z^{k}\right\|_{p}$, to show that (3.5) and (3.6) hold true.

Proof of Theorem 3.5. We use the approach of de Bruijn and Springer [10, 1, 2 , 33] by setting $\Lambda_{n}(z)=n z(1+z)^{n-1}=\sum_{k=0}^{n} k\left(\begin{array}{l}n \\ k\end{array}\right) z^{k}$. This gives $\Lambda P_{n}(z)=$ $z P_{n}^{\prime}(z)$ and $M\left(\Lambda_{n}\right)=n$. Hence (3.7) is a direct consequence of (3.1).

Proof of Corollary 3.6. In order to deduce Corollary 3.6 from Theorem 3.5, we only need to observe that $\left\|z P_{n}^{\prime}\right\|_{0}=\|z\|_{0}\left\|P_{n}^{\prime}\right\|_{0}=\left\|P_{n}^{\prime}\right\|_{0} / \sqrt{e}$.

Proof of Proposition 3.7. For $P_{n}(z)=\sum_{k=0}^{n} a_{k} z^{k}$ we have $P_{n}^{\prime}(z)=\sum_{k=0}^{n} k a_{k} z^{k-1}$, so that

$$
\left\|P_{n}\right\|_{2}^{2}=\sum_{k=0}^{n} \frac{\left|a_{k}\right|^{2}}{k+1} \text { and }\left\|P_{n}^{\prime}\right\|_{2}^{2}=\sum_{k=0}^{n} k\left|a_{k}\right|^{2} .
$$

It follows that

$$
\left\|P_{n}^{\prime}\right\|_{2}^{2}=\sum_{k=0}^{n} k(k+1) \frac{\left|a_{k}\right|^{2}}{k+1} \leq n(n+1) \sum_{k=0}^{n} \frac{\left|a_{k}\right|^{2}}{k+1}=n(n+1)\left\|P_{n}\right\|_{2}^{2} .
$$

The case of equality is verified directly.

\subsection{Proofs for Section 4.}

Proof of Proposition 4.1. Recall that the uniform convergence of $P_{n}$ to $f$ on compact subsets of $\mathbb{D}$ implies that $f$ is analytic in $\mathbb{D}$, and that $P_{n}^{(k)}$ converge to $f^{(k)}$ on compact subsets of $\mathbb{D}$ for any $k \in \mathbb{N}$. In particular,

$$
\lim _{n \rightarrow \infty} P_{n}^{(k)}(0)=f^{(k)}(0) \quad \forall k \geq 0, k \in \mathbb{Z} .
$$

But $P_{n}^{(k)}(0)=k ! a_{k}$, where $a_{k} \in \mathbb{Z}$ is a corresponding coefficient of $P_{n}$. Hence the result follows.

Proof of Proposition 4.2. We have that

$$
\left\|P_{n}-P_{n-1}\right\|_{H^{p}} \leq\left\|f-P_{n}\right\|_{H^{p}}+\left\|f-P_{n-1}\right\|_{H^{p}}
$$

by the triangle inequality for $p \geq 1$, and

$$
\left\|P_{n}-P_{n-1}\right\|_{H^{p}}^{p} \leq\left\|f-P_{n}\right\|_{H^{p}}^{p}+\left\|f-P_{n-1}\right\|_{H^{p}}^{p}
$$


for $0<p<1$. In both cases, (4.1) implies that

$$
\lim _{n \rightarrow \infty}\left\|P_{n}-P_{n-1}\right\|_{H^{p}}=0, \quad 0<p \leq \infty
$$

If $P_{n} \not \equiv P_{n-1}$ then we let $a_{k} z^{k}$ be the lowest nonzero term of $P_{n}-P_{n-1}$, where $\left|a_{k}\right| \in \mathbb{N}$. Using the mean value inequality [12], we obtain

$$
\left\|P_{n}-P_{n-1}\right\|_{H^{p}} \geq\left|a_{k}\right| \geq 1, \quad 0<p \leq \infty .
$$

This is obviously impossible as $n \rightarrow \infty$, so that we have $P_{n} \equiv P_{n-1}$ for all sufficiently large $n \in \mathbb{N}$. Hence the limit function $f$ is also a polynomial with integer coefficients.

Proof of Theorem 4.3. If (4.2) holds then the $P_{n}$ converge to $f$ on compact subsets of $\mathbb{D}$ by the area mean value inequality:

$$
\begin{aligned}
\left|f(z)-P_{n}(z)\right|^{p} & \leq \frac{1}{\pi(1-|z|)^{2}} \iint_{|t-z|<1-|z|}\left|f(t)-P_{n}(t)\right|^{p} d A \\
& \leq \frac{\left\|f(t)-P_{n}(t)\right\|_{p}^{p}}{(1-|z|)^{2}} \rightarrow 0, \quad n \rightarrow \infty, \quad z \in \mathbb{D} .
\end{aligned}
$$

Hence $f$ has a power series expansion at $z=0$ with integer coefficients by Proposition 4.1.

Conversely, suppose that $f \in A^{p}$ is represented by a power series with integer coefficients. Since the partial sums of this series converge to $f$ in $A^{p}$ norm for $1<p<\infty$ by Theorem 4 [12, p. 31], we can select the sequence $P_{n}$ be the sequence of the partial sums.

\subsection{Proofs for Section 5.}

Proof of Proposition 5.1. We apply (2.2) in each variable $z_{j}, j=1, \ldots, d$, and use Fubini's theorem to prove (5.2). Indeed, (2.2) gives that

$$
\begin{aligned}
\frac{1}{2 \pi} \int_{\mathbb{T}} \log \left|P_{n}\left(z_{1}, \ldots, z_{d}\right)\right|\left|d z_{1}\right|-\frac{k_{1}}{2} & \leq \frac{1}{\pi} \int_{\mathbb{D}} \log \left|P_{n}\left(z_{1}, \ldots, z_{d}\right)\right| d A\left(z_{1}\right) \\
& \leq \frac{1}{2 \pi} \int_{\mathbb{T}} \log \left|P_{n}\left(z_{1}, \ldots, z_{d}\right)\right|\left|d z_{1}\right|
\end{aligned}
$$

is true for all $z_{2}, \ldots, z_{d} \in \mathbb{C}$. Integrating the above inequality with respect to $d A\left(z_{2}\right) / \pi$, interchanging the order of integration in the lower and upper 
bounds, and applying (2.2) in the variable $z_{2}$, we obtain

$$
\begin{aligned}
\frac{1}{(2 \pi)^{2}} \int_{\mathbb{T}} \int_{\mathbb{T}} \log \left|P_{n}\left(z_{1}, \ldots, z_{d}\right)\right| & \left|d z_{1}\right|\left|d z_{2}\right|-\frac{k_{1}+k_{2}}{2} \\
& \leq \frac{1}{\pi^{2}} \int_{\mathbb{D}} \int_{\mathbb{D}} \log \left|P_{n}\left(z_{1}, \ldots, z_{d}\right)\right| d A\left(z_{1}\right) d A\left(z_{2}\right) \\
& \leq \frac{1}{(2 \pi)^{2}} \int_{\mathbb{T}} \int_{\mathbb{T}} \log \left|P_{n}\left(z_{1}, \ldots, z_{d}\right)\right|\left|d z_{1}\right|\left|d z_{2}\right|
\end{aligned}
$$

is true for all $z_{3}, \ldots, z_{d} \in \mathbb{C}$. After carrying out this argument for each variable $z_{j}$, we arrive at $(5.2)$ in $d$ steps. When $P_{n}\left(z_{1}, \ldots, z_{d}\right) \neq 0$ in $\mathbb{D}^{d}$, we have that $\left\|P_{n}\right\|_{0}=M\left(P_{n}\right)=\left|a_{0 \ldots 0}\right|$ by the iterative application of Theorem 2.1. If $P_{n}\left(z_{1}, \ldots, z_{d}\right)=a_{k_{1} \ldots k_{d}} z_{1}^{k_{1}} \ldots z_{d}^{k_{d}}$, where $k_{1}+\ldots+k_{d}=n$, then we evaluate directly that $M\left(P_{n}\right)=\left|a_{k_{1} \ldots k_{d}}\right|$ and $\left\|P_{n}\right\|_{0}=\left|a_{k_{1} \ldots k_{d}}\right| e^{-n / 2}$, because $\left\|z_{j}\right\|_{0}=$ $e^{-1 / 2}, j=1, \ldots, n$.

Proof of Example 5.2. (a) Applying (2.1), we have that

$$
\frac{1}{\pi^{2}} \int_{\mathbb{D}} \int_{\mathbb{D}} \log \left|z_{1}+z_{2}\right| d A\left(z_{1}\right) d A\left(z_{2}\right)=\frac{1}{\pi} \int_{\mathbb{D}} \frac{\left|z_{2}\right|^{2}-1}{2} d A\left(z_{2}\right)=-\frac{1}{4} .
$$

(b) is an immediate consequence of (c).

(c) Let $a_{0 \ldots 0}=\left|a_{0 \ldots 0}\right| e^{i \phi}$. Observe that $P_{n}\left(z_{1}, \ldots, z_{d}\right)+\varepsilon e^{i \phi} \neq 0$ in $\mathbb{D}^{d}$ for any $\varepsilon>0$, because

$$
\left|P_{n}\left(z_{1}, \ldots, z_{d}\right)+\varepsilon e^{i \phi}\right| \geq\left|a_{0 \ldots 0}\right|+\varepsilon-\sum_{0<k_{1}+\ldots+k_{d} \leq n}\left|a_{k_{1} \ldots k_{d}}\right|>0
$$

by the triangle inequality. We obtain that $\left\|P_{n}+\varepsilon e^{i \phi}\right\|_{0}=M\left(P_{n}+\varepsilon e^{i \phi}\right)=$ $\left|a_{0 \ldots 0}\right|+\varepsilon$ by the area and contour mean value properties of the (pluri)harmonic function $\log \left|P_{n}\left(z_{1}, \ldots, z_{d}\right)+\varepsilon e^{i \phi}\right|$ in $\mathbb{D}^{d}$, and the result follows by letting $\varepsilon \rightarrow 0$.

\section{REFERENCES}

[1] V. V. Arestov, On integral inequalities for trigonometric polynomials and their derivatives, Math. USSR-Izv. 18 (1982), 1-17.

[2] $\_$Integral inequalities for algebraic polynomials on the unit circle, Math. Notes 48 (1990), 977-984.

[3] A. Baker, Transcendental Number Theory, Cambridge Univ. Press, New York, 1975.

[4] Y. Bilu, Limit distribution of small points on algebraic tori, Duke Math. J. 89 (1997), 465-476.

[5] E. Bombieri, Subvarieties of linear tori and the unit equation: A survey, in "Analytic number theory," ed. by Y. Motohashi, LMS Lecture Notes 247 (1997), Cambridge Univ. Press, Cambridge, pp. 1-20.

[6] P. Borwein, Computational Excursions in Analysis and Number Theory, SpringerVerlag, New York, 2002. 
[7] P. Borwein and T. Erdélyi, Polynomials and Polynomial Inequalities, Springer-Verlag, New York, 1995.

[8] D. W. Boyd, Variations on a theme of Kronecker, Canad. Math. Bull. 21 (1978), 1244-1260.

[9] _ Speculations concerning the range of Mahler measure, Canad. Math. Bull. 24 (1981), 453-469.

[10] N. G. de Bruijn and T. A. Springer, On the zeros of composition-polynomials, Indag. Math. 9 (1947), 406-414.

[11] A. Dubickas and C. J. Smyth, The Lehmer constants of an annulus, J. Théor. Nombres Bordeaux 13 (2001), 413-420.

[12] P. L. Duren and A. Schuster, Bergman Spaces, American Mathematical Society, Providence, 2004.

[13] P. Erdős and P. Turán, On the distribution of roots of polynomials, Ann. of Math. 51 (1950), 105-119.

[14] G. Everest and T. Ward, Heights of Polynomials and Entropy in Algebraic Dynamics, Springer-Verlag, London, 1999.

[15] M. Fekete, Über die Verteilung der Wurzeln bei gewissen algebraischen Gleichungen mit ganzzahligen Koeffizienten, Math. Zeit. 17 (1923), 228-249.

[16] Le Baron O. Ferguson, Approximation by Polynomials with Integral Coefficients, Amer. Math. Soc., Providence, R.I., 1980.

[17] E. Ghate and E. Hironaka, The arithmetic and geometry of Salem numbers, Bull. Amer. Math. Soc. 38 (2001), 293-314.

[18] G. H. Hardy, J. E. Littlewood and G. Pólya, Inequalities, Cambridge Univ. Press, London, 1952.

[19] H. Hedenmalm, B. Korenblum and K. Zhu, Theory of Bergman Spaces, SpringerVerlag, New York, 2000.

[20] R. Jentzsch, Untersuchungen zur Theorie der Folgen analytischer Funktionen, Acta Math. 41 (1917), 219-270.

[21] L. Kronecker, Zwei Sätze über Gleichungen mit ganzzahligen Koeffizienten, J. Reine Angew. Math. 53 (1857), 173-175.

[22] M. Langevin, Méthode de Fekete-Szegö et problème de Lehmer, C. R. Acad. Sci. Paris Sér. I Math. 301 (1985), 463-466.

[23] _ Minorations de la maison et de la mesure de Mahler de certains entiers algébriques, C. R. Acad. Sci. Paris Sér. I Math. 303 (1986), 523-526.

[24] _ Calculs explicites de constantes de Lehmer, Groupe de travail en théorie analytique et élémentaire des nombres, 1986-1987, 52-68, Publ. Math. Orsay, 88-01, Univ. Paris XI, Orsay, 1988.

[25] D. H. Lehmer, Factorization of certain cyclotomic functions, Ann. of Math. 34 (1933), 461-479.

[26] K. Mahler, On the zeros of the derivative of a polynomial, Proc. Roy. Soc. London Ser. A 264 (1961), 145-154.

[27] _ On two extremum properties of polynomials, Illinois J. Math. 7 (1963), 681701.

[28] _ A remark on a paper of mine on polynomials, Illinois J. Math. 8 (1964), 1-4.

[29] A. Máté and P. Nevai, Bernstein inequality in $L^{p}$ for $0<p<1$ and $(C, 1)$ bounds of orthogonal polynomials, Ann. of Math. 111 (1980), 145-154.

[30] M. Mignotte, Sur un théorème de M. Langevin, Acta Arith. 54 (1989), 81-86.

[31] G. V. Milovanović, D. S. Mitrinović and Th. M. Rassias, Topics in Polynomials: Extremal Problems, Inequalities, Zeros, World Scientific, Singapore, 1994.

[32] I. E. Pritsker, An areal analog of Mahler's measure, Illinois J. Math. (to appear) 
[33] Q. I. Rahman and G. Schmeisser, Analytic Theory of Polynomials, Clarendon Press, Oxford, 2002.

[34] T. Ransford, Potential Theory in the Complex Plane, Cambridge University Press, Cambridge, 1995.

[35] G. Rhin and C. J. Smyth, On the absolute Mahler measure of polynomials having all zeros in a sector, Math. Comp. 65 (1995), 295-304.

[36] R. Rumely, On Bilu's equidistribution theorem, in "Spectral problems in geometry and arithmetic (Iowa City, IA, 1997)," Contemp. Math. 237, Amer. Math. Soc., Providence, RI, 1999, pp. 159-166.

[37] A. Schinzel, On the product of the conjugates outside the unit circle of an algebraic number, Acta Arith. 24 (1973), 385-399. (Addendum: Acta Arith. 26 (1974/75), 329331.)

[38] T. Sheil-Small, Complex Polynomials, Cambridge University Press, Cambridge, 2002.

[39] C. J. Smyth, On measures of polynomials in several variables, Bull. Australian Math. Soc. 23 (1981), 49-63. (Corrigendum: G. Myerson and C. J. Smyth, Bull. Austral. Math. Soc. 26 (1982), 317-319.)

[40] _ A Kronecker-type theorem for complex polynomials in several variables, Canad. Math. Bull. 24 (1981), 447-452. (Addenda and errata: Canad. Math. Bull. 25 (1982), 504.)

[41] _ The Mahler measure of algebraic numbers: A survey, this volume.

[42] E. A. Storozhenko, A problem of Mahler on the zeros of a polynomial and its derivative, Sb. Math. 187 (1996), 735-744.

[43] G. Szegö, Beiträge zur Theorie der Toeplitzschen Formen, I, Math. Zeit. 6 (1920), 167-202.

[44] _ _ Über die Nullstellen von Polynomen, die in einem Kreis gleichmässig konvergieren, Sitzungsber. Ber. Math. Ges. 21 (1922), 59-64.

[45] R. M. Trigub, Approximation of functions with Diophantine conditions by polynomials with integral coefficients, in "Metric Questions of the Theory of Functions and Mappings," No. 2, Naukova Dumka, Kiev, 1971, pp. 267-333. (Russian)

[46] M. Tsuji, Potential Theory in Modern Function Theory, Chelsea Publ. Co., New York, 1975.

[47] A. Zygmund, A remark on conjugate series, Proc. London Math. Soc. 34 (1932), 392400 . 